\title{
Nonlinear biharmonic boundary value problem
}

\author{
Tacksun Jung ${ }^{1}$ and Q-Heung Choi ${ }^{2 *}$
}

${ }^{*}$ Correspondence: qheung@inha.ac.kr

${ }^{2}$ Department of Mathematics Education, Inha University, Incheon, 402-751, Korea

Full list of author information is available at the end of the article

\begin{abstract}
We consider the nonlinear biharmonic equation with variable coefficient and polynomial growth nonlinearity and Dirichlet boundary condition. We get two theorems. One theorem says that there exists at least one bounded solution under some condition. The other one says that there exist at least two solutions, one of which is a bounded solution and the other of which has a large norm under some condition. We obtain this result by the variational method, generalized mountain pass geometry and the critical point theory of the associated functional.
\end{abstract}

MSC: 35J20; 35J25; 35Q72

Keywords: biharmonic boundary value problem; polynomial growth; variational method; generalized mountain pass geometry; critical point theory; (PS) condition

\section{Introduction}

Let $\Omega$ be a bounded domain in $R^{n}$ with smooth boundary $\partial \Omega$ and $L^{2}(\Omega)$ be a square integrable function space defined on $\Omega$. Let $\Delta$ be the elliptic operator and $\Delta^{2}$ be the biharmonic operator. Let $c \in R$. In this paper we study the following nonlinear biharmonic equation with Dirichlet boundary condition:

$$
\begin{aligned}
& \Delta^{2} u+c \Delta u=a(x) g(u) \quad \text { in } \Omega, \\
& u=0, \quad \Delta u=0 \quad \text { on } \partial \Omega,
\end{aligned}
$$

where $a: \bar{\Omega} \rightarrow R$ is a continuous function which changes sign in $\Omega$.

We assume that $g$ satisfies the following conditions:

(g1) $g \in C(R, R)$,

(g2) there are constants $a_{1}, a_{2} \geq 0$ such that

$$
|g(u)| \leq a_{1}+a_{2}|u|^{\mu-1},
$$

where $2<\mu<\frac{2 n}{n-2}$ if $n \geq 3$,

(g3) there exists a constant $r_{0} \geq 0$ such that

$$
0<\mu G(\xi)=\mu \int_{0}^{\xi} g(t) d t \leq \xi g(\xi) \text { for }|\xi| \geq r_{0}
$$

(g4) $g(u)=o(|u|)$ as $u \rightarrow 0$. 
We note that (g3) implies the existence of the positive constants $a_{3}, a_{4}, a_{5}$ such that

$$
\frac{1}{\mu}\left(\xi g(\xi)+a_{3}\right) \geq G(\xi)+a_{4} \geq a_{5}|\xi|^{\mu} \quad \text { for } \xi \in R
$$

Remark 1.1 The real number $\xi$ in the definition (g3) is not automatically nonnegative. The reason is as follows.

Since $0<\mu G(\xi)<\xi g(\xi)$ and $\mu>2, G(\xi)>0$ and $\xi g(\xi)>0$. By $\xi g(\xi)>0$, we have two cases: one case is that $\xi>0$ and $g(\xi)>0$. The other case is that $\xi<0$ and $g(\xi)<0$. Thus $\xi$ is not nonnegative.

Remark 1.2 We obtain the boundedness of $\frac{1}{2} g(u) u-G(u)$ as follows.

By the condition (g3), $\frac{1}{2} g(u) u \geq \frac{1}{2} \mu G(u)$ for $|u| \geq r_{0}$. Since $\mu>2, \frac{1}{2}-\frac{1}{\mu}>0$, and $G(u)+$ $a_{4} \geq a_{5}|u|^{\mu}$ in (1.2),

$$
\begin{aligned}
\frac{1}{2} g(u) u-G(u) & \geq \frac{1}{2} \mu G(u)-G(u) \\
& =\mu\left(\frac{1}{2}-\frac{1}{\mu}\right) G(u) \\
& \geq \mu\left(\frac{1}{2}-\frac{1}{\mu}\right)\left(a_{5}|u|^{\mu}-a_{4}\right) .
\end{aligned}
$$

Thus we obtain the boundedness of $\frac{1}{2} g(u) u-G(u)$.

Remark 1.3 (i) Assumption (g4) implies that (1.1) has a trivial solution.

(ii) If $n=1$, (g2) can be dropped. If $n=2$, it suffices that

$$
|g(u)| \leq a_{1} \exp p(\xi)
$$

where $p(\xi) \xi^{-2} \rightarrow 0$ as $|\xi| \rightarrow \infty$.

(iii) If $n \geq 3$ and $g(\xi)=\xi^{+1+\epsilon}$, where $\xi^{+}=\max \{\xi$, 0$\}$ and $\epsilon>0$ is a small number, then (g1)-(g4) are satisfied.

The eigenvalue problem

$$
\begin{aligned}
& \Delta u+\lambda u=0 \quad \text { in } \Omega, \\
& u=0 \quad \text { on } \partial \Omega
\end{aligned}
$$

has infinitely many eigenvalues $\lambda_{k}, k \geq 1$, and corresponding eigenfunctions $\phi_{k}, k \geq 1$, suitably normalized with respect to the $L^{2}(\Omega)$ inner product, where each eigenvalue $\lambda_{k}$ is repeated as often as its multiplicity. The eigenvalue problem

$$
\begin{aligned}
& \Delta^{2} u+c \Delta u=\Lambda u \quad \text { in } \Omega, \\
& u=0, \quad \Delta u=0 \quad \text { on } \partial \Omega
\end{aligned}
$$

has also infinitely many eigenvalues $\lambda_{k}\left(\lambda_{k}-c\right), k \geq 1$ and corresponding eigenfunctions $\phi_{k}, k \geq 1$. We note that $\lambda_{1}\left(\lambda_{1}-c\right) \leq \lambda_{2}\left(\lambda_{2}-c\right) \leq \cdots \rightarrow+\infty$, and that $\phi_{1}(x)>0$ for $x \in \Omega$. 
Khanfir and Lassoued [1] showed the existence of at least one solution for the nonlinear elliptic boundary problem when $g$ is locally Hölder continuous on $R_{+}$. Choi and Jung [2] showed that the problem

$$
\begin{aligned}
& \Delta^{2} u+c \Delta u=b u^{+}+s \quad \text { in } \Omega, \\
& u=0, \quad \Delta u=0 \quad \text { on } \partial \Omega
\end{aligned}
$$

has at least two nontrivial solutions when $\left(c<\lambda_{1}, \lambda_{1}\left(\lambda_{1}-c\right)<b<\lambda_{2}\left(\lambda_{2}-c\right)\right.$ and $\left.s<0\right)$ or $\left(\lambda_{1}<c<\lambda_{2}, b<\lambda_{1}\left(\lambda_{1}-c\right)\right.$ and $\left.s>0\right)$. The authors obtained these results by using the variational reduction method. The authors [3] also proved that when $c<\lambda_{1}, \lambda_{1}\left(\lambda_{1}-c\right)<$ $b<\lambda_{2}\left(\lambda_{2}-c\right)$ and $s<0,(1.3)$ has at least three nontrivial solutions by using degree theory. Tarantello [4] also studied

$$
\begin{aligned}
& \Delta^{2} u+c \Delta u=b\left((u+1)^{+}-1\right), \\
& u=0, \quad \Delta u=0 \quad \text { on } \partial \Omega .
\end{aligned}
$$

She showed that if $c<\lambda_{1}$ and $b \geq \lambda_{1}\left(\lambda_{1}-c\right)$, then (1.4) has a negative solution. She obtained this result by degree theory. Micheletti and Pistoia [5] also proved that if $c<\lambda_{1}$ and $b \geq$ $\lambda_{2}\left(\lambda_{2}-c\right)$ then (1.4) has at least four solutions by the variational linking theorem and Leray-Schauder degree theory.

In this paper we are trying to find the weak solutions of (1.1), that is,

$$
\int_{\Omega}\left(\Delta^{2} u+c \Delta u-a(x) g(u)\right) v d x=0 \quad \text { for any } v \in H,
$$

where the space $H$ is introduced in Section 2. Let us set

$$
\Omega^{+}=\{x \in \Omega \mid a(x)>0\}, \quad \Omega^{-}=\{x \in \Omega \mid a(x)<0\}
$$

and let

$$
a^{+}=a \cdot \chi_{\Omega^{+}}, \quad a^{-}=-a \cdot \chi_{\Omega^{-}} .
$$

Since $a(x)$ changes sign, the open subsets $\Omega^{+}$and $\Omega^{-}$are nonempty. Now we can write $a=a^{+}-a^{-}$. Our main results are as follows.

Theorem 1.1 Assume that $\lambda_{k}<c<\lambda_{k+1}$, g satisfies (g1)-(g4) and $g(u) u-\mu G(u)$ is bounded. Then (1.1) has at least one bounded nontrivial solution.

Theorem 1.2 Assume that $\lambda_{k}<c<\lambda_{k+1}, g$ satisfies (g1)-(g4), $g(u) u-\mu G(u)$ is not bounded and there exists a small $\epsilon>0$ such that $\int_{\Omega^{-}} a^{-}(x) d x<\epsilon$. Then (1.1) has at least two solutions, (i) one of which is nontrivial and bounded, and (ii) the other of which has a large norm such that

$$
\max _{x \in \Omega}|u(x)|>M \quad \text { for some } M
$$

The outline of Theorem 1.1 and Theorem 1.2 is as follows: In Section 2, we prove that the corresponding functional $I(u)$ of $(1.1)$, which is introduced in (2.1), is continuous and 
Fréchet differentiable and satisfies the (PS) condition. In Section 3, we prove Theorem 1.1. In Section 4, we prove Theorem 1.2 by the variational method, the generalized mountain pass geometry and the critical point theory.

\section{Palais-Smale condition}

Any element $u$ in $L^{2}(\Omega)$ can be written as

$$
u=\sum h_{k} \phi_{k} \text { with } \sum h_{k}^{2}<\infty
$$

We define a subspace $H$ of $L^{2}(\Omega)$ as follows:

$$
H=\left\{u \in L^{2}(\Omega)\left|\sum\right| \lambda_{k}\left(\lambda_{k}-c\right) \mid h_{k}^{2}<\infty\right\} .
$$

Then this is a Banach space with a norm

$$
\|u\|=\left[\sum\left|\lambda_{k}\left(\lambda_{k}-c\right)\right| h_{k}^{2}\right]^{\frac{1}{2}}
$$

Since $\lambda_{k} \rightarrow+\infty$ and $c$ is fixed, we have

(i) $\Delta^{2} u+c \Delta u \in H$ implies $u \in H$,

(ii) $\|u\| \geq C\|u\|_{L^{2}(\Omega)}$, for some $C>0$,

(iii) $\|u\|_{L^{2}(\Omega)}=0$ if and only if $\|u\|=0$,

which are proved in [6].

Let

$$
\begin{aligned}
& H_{+}=\left\{u \in H \mid h_{k}=0 \text { if } \lambda_{k}\left(\lambda_{k}-c\right)<0\right\}, \\
& H_{-}=\left\{u \in H \mid h_{k}=0 \text { if } \lambda_{k}\left(\lambda_{k}-c\right)>0\right\} .
\end{aligned}
$$

Then $H=H_{-} \oplus H_{+}$. Let $P_{+}$be the orthogonal projection on $H_{+}$and $P_{-}$be the orthogonal projection on $H_{-}$.

We are looking for the weak solutions of (1.1). The weak solutions of (1.1) coincide with the critical points of the associated functional

$$
\begin{aligned}
I(u) & \in C^{1}(H, R), \\
I(u) & =\int_{\Omega}\left[\frac{1}{2}|\Delta u|^{2}-\frac{c}{2}|\nabla u|^{2}-\int_{\Omega} a(x) G(u)\right] d x \\
& =\frac{1}{2}\left(\left\|P_{+} u\right\|^{2}-\left\|P_{-} u\right\|^{2}\right)-\int_{\Omega} a(x) G(u) d x .
\end{aligned}
$$

By (g1) and (g2), $I$ is well defined. By Proposition 2.1, $I \in C^{1}(H, R)$ and $I$ is Fréchet differentiable in $H$.

Proposition 2.1 Assume that $\lambda_{k}<c<\lambda_{k+1}, k \geq 1$, and that $g$ satisfies (g1)-(g4). Then I(u) is continuous and Fréchet differentiable in $H$ with Fréchet derivative

$$
I^{\prime}(u) h=\int_{\Omega}[\Delta u \cdot \Delta h-c \nabla u \cdot \nabla h-a(x) g(u) h] d x .
$$


If we set

$$
K(u)=\int_{\Omega} a(x) G(u) d x,
$$

then $K^{\prime}(u)$ is continuous with respect to weak convergence, $K^{\prime}(u)$ is compact, and

$$
K^{\prime}(u) h=\int_{\Omega} a(x) g(u) h d x \quad \text { for all } h \in H .
$$

This implies that $I \in C^{1}(H, R)$ and $K(u)$ is weakly continuous.

The proof of Proposition 2.1 is the same as that of Appendix B in [7].

Proposition 2.2 (Palais-Smale condition) Assume that $\lambda_{k}<c<\lambda_{k+1}, k \geq 1$, and g satisfies (g1)-(g4). We also assume that $g(u) u-\mu G(u)$ is bounded or that there exists an $\epsilon>0$ such that $\int_{\Omega^{-}} a^{-}(x) d x<\epsilon$. Then $I(u)$ satisfies the Palais-Smale condition.

Proof Suppose that $\left(u_{m}\right)$ is a sequence with $I\left(u_{m}\right) \leq M$ and $I^{\prime}\left(u_{m}\right) \rightarrow 0$ as $m \rightarrow \infty$. Then by (g2), (g3), and the Hölder inequality and the Sobolev Embedding Theorem, for large $m$ and $\mu>2$ with $u=u_{m}$, we have

$$
\begin{aligned}
M+\frac{1}{2}\|u\| \geq & I(u)-\frac{1}{2} I^{\prime}(u) u=\int_{\Omega}\left[\frac{1}{2} a(x) g(u) u-a(x) G(u)\right] d x \\
= & \int_{\Omega} a^{+}(x)\left[\frac{1}{2} g(u) u-G(u)\right] d x-\int_{\Omega} a^{-}(x)\left[\frac{1}{2} g(u) u-G(u)\right] d x \\
\geq & \left(\frac{1}{2}-\frac{1}{\mu}\right) \mu \int_{\Omega} a^{+}(x) \cdot G(u) d x-\max _{\Omega}\left|\frac{1}{2} g(u) u-G(u)\right| \int_{\Omega^{-}} a^{-}(x) d x \\
\geq & \left(\frac{1}{2}-\frac{1}{\mu}\right) \mu \int_{\Omega} a^{+}(x) \cdot\left(a_{5}|u|^{\mu}-a_{4}\right) d x \\
& -\max _{\Omega}\left|\frac{1}{2} g(u) u-G(u)\right| \int_{\Omega^{-}} a^{-}(x) d x .
\end{aligned}
$$

Since $\frac{1}{2} g(u) u-G(u)$ is bounded or there exists an $\epsilon>0$ such that $\int_{\Omega^{-}} a^{-}(x)<\epsilon$; we have

$$
1+\|u\| \geq M_{1} \int_{\Omega}|u|^{\mu} d x \geq M_{2}\left(\int_{\Omega}|u|^{2} d x\right)^{\frac{1}{2} \cdot \mu} .
$$

Moreover since

$$
\left|I^{\prime}\left(u_{m}\right) \varphi\right| \leq\|\varphi\|
$$

for large $m$ and all $\varphi \in H$, choosing $\varphi=P_{+} u_{m} \in H_{+}$gives

$$
\begin{aligned}
\left\|P_{+} u_{m}\right\|^{2} & =\int_{\Omega}\left(\Delta^{2} u_{m}+c \Delta u_{m}\right) \cdot P_{+} u_{m} d x \\
& =\int_{\Omega} a(x) g\left(u_{m}\right) P_{+} u_{m} d x \\
& \leq \int_{\Omega}|a(x)|\left|g\left(u_{m}\right)\right|\left|u_{m}\right| d x
\end{aligned}
$$




$$
\begin{aligned}
& \leq\|a\|_{\infty} \int_{\Omega}\left(a_{1}\left|u_{m}\right|+a_{2}\left|u_{m}\right|^{\mu}\right) d x \\
& \leq C_{1} \int_{\Omega}\left|u_{m}\right|^{\mu} d x+C_{2}\left\|u_{m}\right\|_{L^{2}(\Omega)} \\
& \leq C_{1} \int_{\Omega}\left|u_{m}\right|^{\mu} d x+C_{2}^{\prime}\left\|u_{m}\right\| .
\end{aligned}
$$

Taking $\varphi=-P_{-} u_{m}$ in (2.4) yields

$$
\begin{aligned}
\left\|P_{-} u_{m}\right\|^{2} & =\int_{\Omega}\left(\Delta^{2} u_{m}+c \Delta u_{m}\right) \cdot\left(-P_{-} u_{m}\right) d x \\
& =\int_{\Omega} a(x) g\left(u_{m}\right) \cdot\left(-P_{-} u_{m}\right) d x \\
& \leq \int_{\Omega}|a(x)|\left|g\left(u_{m}\right)\right|\left|u_{m}\right| d x \\
& \leq C_{3} \int_{\Omega}\left|u_{m}\right|^{\mu} d x+C_{4}\left\|u_{m}\right\| .
\end{aligned}
$$

Thus, by (2.3), we have

$$
\begin{aligned}
\left\|u_{m}\right\|^{2} & =\left\|P_{+} u_{m}\right\|^{2}+\left\|P_{-} u_{m}\right\|^{2} \leq M_{3} \int_{\Omega}\left|u_{m}\right|^{\mu} d x+M_{4}\left\|u_{m}\right\| \\
& \leq M_{5}\left(1+\left\|u_{m}\right\|\right)+M_{4}\left\|u_{m}\right\| \leq M_{6}\left(1+\left\|u_{m}\right\|\right)
\end{aligned}
$$

from which the boundedness of $\left(u_{m}\right)$ follows. Thus $\left(u_{m}\right)$ converges weakly in $H$. Since $P_{ \pm} I^{\prime}\left(u_{m}\right)= \pm P_{ \pm} u_{m}+P_{ \pm} \tilde{\mathcal{P}}\left(u_{m}\right)$ with $\tilde{\mathcal{P}}$ compact and the weak convergence of $P_{ \pm} u_{m}$ imply the strong convergence of $P_{ \pm} u_{m}$ and hence (PS) condition holds.

\section{Proof of Theorem 1.1}

We shall show that $I(u)$ satisfies the generalized mountain pass geometrical assumptions.

We recall the generalized mountain pass geometry.

Let $H=V \oplus X$, where $V \neq\{0\}$ and is finite dimensional. Suppose that $I \in C^{1}(H, R)$, satisfies the Palais-Smale condition, and

(i) there are constants $\rho, \alpha>0$ and a bounded neighborhood $B_{\rho}$ of 0 such that

$$
\left.I\right|_{\partial B_{\rho} \cap X} \geq \alpha \text {, and }
$$

(ii) there is an $e \in \partial B_{1} \cap X$ and $R>\rho$ such that if $Q=\left(\bar{B}_{R} \cap V\right) \oplus\{r e \mid 0<r<R\}$, then

$$
\left.I\right|_{\partial Q} \leq 0 \text {. }
$$

Then $I$ possesses a critical value $b \geq \alpha$. Moreover $b$ can be characterized as

$$
b=\inf _{\gamma \in \Gamma} \max _{u \in Q} I(\gamma(u)),
$$

where

$$
\Gamma=\{\gamma \in C(\bar{Q}, H) \mid \gamma=i d \text { on } \partial Q\}
$$

Let $H_{k}=\operatorname{span}\left\{\phi_{1}, \ldots, \phi_{k}\right\}$. Then $H_{k}$ is a subspace of $H$ such that

$$
H=\bigoplus_{k \in N} H_{k} \quad \text { and } \quad H=H_{k} \oplus H_{k}^{\perp} \text {. }
$$


Let

$$
B_{r}=\{u \in H \mid\|u\| \leq r\} .
$$

We have the following generalized mountain pass geometrical assumptions.

Lemma 3.1 Assume that $\lambda_{k}<c<\lambda_{k+1}$ and $g$ satisfies (g1)-(g4). Then

(i) there are constants $\rho>0, \alpha>0$ and a bounded neighborhood $B_{\rho}$ of 0 such that $\left.I\right|_{\partial B_{\rho} \cap H_{k}^{\perp}} \geq \alpha$, and

(ii) there is an $e \in \partial B_{1} \cap H_{k}^{\perp}$ and $R>\rho$ such that if $Q=\left(\bar{B}_{R} \cap H_{k}\right) \oplus\{r e \mid 0<r<R\}$, then $\left.I\right|_{\partial Q} \leq 0$, and

(iii) there exists $u_{0} \in H$ such that $\left\|u_{0}\right\|>\rho$ and $I\left(u_{0}\right) \leq 0$.

Proof (i) Let $u \in H_{k}^{\perp}$. Then

$$
\int_{\Omega}\left(\Delta^{2} u+c \Delta u\right) u d x \geq \lambda_{k+1}\left(\lambda_{k+1}-c\right)\|u\|_{L^{2}(\Omega)}^{2}>0 .
$$

Thus by (g2), (g4), and the Hölder inequality, we have

$$
\begin{aligned}
I(u) & =\frac{1}{2}\left\|P_{+} u\right\|^{2}-\frac{1}{2}\left\|P_{-} u\right\|^{2}-\int_{\Omega} a(x) G(u) d x \\
& \geq \frac{1}{2}\left\|P_{+} u\right\|^{2}-\|a\|_{\infty} \int_{\Omega} C_{1}|u|^{\mu} d x \\
& \geq \frac{1}{2}\left\|P_{+} u\right\|^{2}-\|a\|_{\infty} C_{1}^{\prime}\|u\|^{\mu}
\end{aligned}
$$

for $C_{1}, C_{1}^{\prime}>0$. Since $\mu>2$, there exist $\rho>0$ and $\alpha>0$ such that if $u \in \partial B_{\rho}$, then $I(u) \geq \alpha$.

(ii) Let $B_{r}$ be a ball with radius $r>0$, $e$ be a fixed element in $\partial B_{1} \cap H_{k}^{\perp}$ and $u \in\left(\bar{B}_{r} \cap H_{k}\right) \oplus$ $\{r e \mid 0<r\}$. Then $u=v+w, v \in B_{r} \cap H_{k}, w=r e$. We note that

$$
\text { since } v \in H_{k}, \quad \int_{\Omega}\left(\Delta^{2} v+c \Delta v\right) v d x \leq \lambda_{k}\left(\lambda_{k}-c\right)\|v\|_{L^{2}(\Omega)}^{2}<0 \text {. }
$$

Thus we have

$$
\begin{aligned}
I(u) & =\frac{1}{2} r^{2}-\frac{1}{2}\left\|P_{-} v\right\|^{2}-\int_{\Omega} a(x) G(v+r e) d x \\
& \leq \frac{1}{2} r^{2}+\frac{1}{2}\left(\lambda_{k}\left(\lambda_{k}-c\right)\right)\|v\|_{L^{2}(\Omega)}^{2}-\int_{\Omega^{+}} a(x)\left(a_{5}|v+r e|^{\mu}-a_{4}\right) d x .
\end{aligned}
$$

Since $\mu>2$, there exists $R>0$ such that if $u \in Q=\left(\bar{B}_{R} \cap H_{k}\right) \oplus\{r e \mid 0<r<R\}$, then $I(u)<0$.

(iii) If we choose $\psi \in H$ such that $\|\psi\|=1, \psi \geq 0$ in $\Omega$ and $\operatorname{supp}(\psi) \subset \Omega^{+}$, then we have

$$
\begin{aligned}
I(t \psi) & \leq \frac{1}{2}\left\|P_{+}(t \psi)\right\|^{2}-\frac{1}{2}\left\|P_{-}(t \psi)\right\|^{2}-\int_{\Omega^{+}} a(x)\left(a_{5} t^{\mu} \psi^{\mu}-a_{4}\right) d x \\
& \leq \frac{1}{2}\|t \psi\|^{2}-\int_{\Omega^{+}} a(x)\left(a_{5} t^{\mu} \psi^{\mu}-a_{4}\right) d x \\
& =\frac{1}{2} t^{2}-\int_{\Omega^{+}} a(x)\left(a_{5} t^{\mu} \psi^{\mu}-a_{4}\right) d x
\end{aligned}
$$


for all $t>0$. Since $\mu>2$, for $t_{0}$ great enough, $u_{0}=t_{0} \psi$ is such that $\left\|u_{0}\right\|>\rho$ and $I\left(u_{0}\right) \leq 0$.

Proof of Theorem 1.1 By Proposition 2.1 and Proposition 2.2, $I(u) \in C^{1}(H, R)$ and satisfies the Palais-Smale condition. By Lemma 3.1, there are constants $\rho>0, \alpha>0$ and a bounded neighborhood $B_{\rho}$ of 0 such that $\left.I\right|_{\partial B_{\rho} \cap H_{m}^{\perp}} \geq \alpha$, and there is an $e \in \partial B_{1} \cap H_{k}^{\perp}$ and $R>\rho$ such that if $Q=\left(\bar{B}_{R} \cap H_{k}\right) \oplus\{r e \mid 0<r<R\}$, then $\left.I\right|_{\partial Q} \leq 0$, and there exists $u_{0} \in H$ such that $\left\|u_{0}\right\|>\rho$ and $I\left(u_{0}\right) \leq 0$. By the generalized mountain pass theorem, $I(u)$ has a critical value $b \geq \alpha$. Moreover, $b$ can be characterized as

$$
b=\inf _{\gamma \in \Gamma} \max _{u \in Q} I(\gamma(u))
$$

where

$$
\Gamma=\{\gamma \in C(\bar{Q}, H) \mid \gamma=i d \text { on } \partial Q\} .
$$

We denote by $\tilde{u}$ a critical point of $I$ such that $I(\tilde{u})=b$. We claim that there exists a constant $C>0$ such that

$$
\left\|a^{+}(x)^{\frac{1}{\mu}} \tilde{u}\right\|_{L^{2}(\Omega)} \leq C\left(1+L \int_{\Omega^{-}} a^{-}(x) d x\right)^{\frac{1}{\mu}}
$$

where $L=\max _{\Omega}\left|\frac{1}{2} g(\tilde{u}) \tilde{u}-G(\tilde{u})\right|$.

In fact, we have

$$
b \leq \max _{0 \leq t \leq 1} I\left(t u_{0}\right)
$$

and

$$
\begin{aligned}
I\left(t u_{0}\right) & =t^{2}\left(\frac{1}{2}\left\|P_{+} u_{0}\right\|^{2}-\frac{1}{2}\left\|P_{-} u_{0}\right\|^{2}\right)-\int_{\Omega} a(x) G\left(t u_{0}\right) d x \\
& \leq t^{2}\left\|u_{0}\right\|^{2}-\int_{\Omega} a^{+}(x) G\left(t u_{0}\right) d x+\int_{\Omega} a^{-}(x) G\left(t u_{0}\right) d x \\
& \leq t^{2}\left\|u_{0}\right\|^{2}-a_{5} t^{\mu} \int_{\Omega} a^{+}(x) u_{0}^{\mu} d x+a_{4} \int_{\Omega} a^{+}(x) d x+a_{5} t^{\mu} \int_{\Omega} a^{-}(x) u_{0}^{\mu} d x \\
& =C t^{2}-C t^{\mu}+C+C^{\prime} t^{\mu} .
\end{aligned}
$$

Since $0 \leq t \leq 1, b$ is bounded: $b<\tilde{C}$.

We can write

$$
\begin{aligned}
b & =I(\tilde{u})-\frac{1}{2} I^{\prime}(\tilde{u}) \tilde{u} \\
& =\int_{\Omega} a(x)\left(\frac{1}{2} g(\tilde{u}) \tilde{u}-G(\tilde{u})\right) d x \\
& =\int_{\Omega} a^{+}(x)\left(\frac{1}{2} g(\tilde{u}) \tilde{u}-G(\tilde{u})\right) d x-\int_{\Omega} a^{-}(x)\left(\frac{1}{2} g(\tilde{u}) \tilde{u}-G(\tilde{u})\right) d x
\end{aligned}
$$




$$
\begin{aligned}
& \geq\left(\frac{1}{2}-\frac{1}{\mu}\right) \int_{\Omega} a^{+}(x) g(\tilde{u}) \tilde{u} d x-\max _{\Omega}\left|\frac{1}{2} g(\tilde{u}) \tilde{u}-G(\tilde{u})\right| \int_{\Omega^{-}} a^{-}(x) d x \\
& \geq\left(\frac{1}{2}-\frac{1}{\mu}\right) \mu \int_{\Omega} a^{+}(x)\left(a_{3}|\tilde{u}|^{\mu}-a_{4}\right) d x-L \int_{\Omega^{-}} a^{-}(x) d x,
\end{aligned}
$$

where $L=\max _{\Omega}\left|\frac{1}{2} g(\tilde{u}) \tilde{u}-G(\tilde{u})\right|$. Thus we have

$$
C^{\prime}\left(1+L \int_{\Omega^{-}} a^{-}(x) d x\right) \geq \int_{\Omega} a^{+}(x)|\tilde{u}|^{\mu} d x \geq C^{\prime \prime}\left[\int_{\Omega}\left(a^{+}(x)^{\frac{1}{\mu}}|\tilde{u}|\right)^{2} d x\right]^{\frac{\mu}{2}}
$$

for some constants $C^{\prime}, C^{\prime \prime}>0$, from which we conclude that $\tilde{u}$ is bounded and the proof of Theorem 1.1 is complete.

\section{Proof of Theorem 1.2}

Assume that $\frac{1}{2} g(u) u-G(u)$ is not bounded and there exists an $\epsilon>0$ such that $\int_{\Omega^{-}} a^{-}(x$, $t) d x<\epsilon$. By Proposition 2.1 and Proposition 2.2, $I \in C^{1}(H, R)$ and satisfies the Palais-Smale condition. By Lemma 3.1 and the generalized mountain pass theorem, $I(u)$ has a critical value $b$ with critical point $\tilde{u}$ such that $I(\tilde{u})=b$. If $\int_{\Omega^{-}} a^{-}(x) d x$ is sufficiently small, by (3.1), we have

$$
\int_{\Omega} a^{+}(x)|\tilde{u}|^{\mu} d x \leq C
$$

for $C>0$, from which we can conclude that $\tilde{u}$ is bounded and the proof of Theorem 1.2(i) is complete.

Next we shall prove Theorem 1.2(ii). We may assume that $R_{n}<R_{n+1}$ for all $n \in N$. Let us set $D_{n}=B_{R_{n}} \cap H_{n}, \partial D_{n}=\partial B_{R_{n}} \cap H_{n}$.

Lemma 4.1 Assume that $g$ satisfies (g1)-(g4), $\frac{1}{2} g(u) u-G(u)$ is not bounded and there exists an $\epsilon>0$ such that $\int_{\Omega^{-}} a^{-}(x, t)<\epsilon$. Then there exists an $R_{n}>0$ such that

$$
I(u) \leq 0 \quad \text { for } u \in H_{n} \backslash B_{R_{n}} .
$$

Proof Let us choose $\psi \in H$ such that $\|\psi\|=1, \psi \geq 0$ in $\Omega$ and $\operatorname{supp}(\psi) \subset \Omega^{+}$. Then, by (g2), (g4), and the Hölder inequality, we have

$$
\begin{aligned}
I(t \psi)= & \frac{1}{2}\left\|P_{+} t \psi\right\|^{2}-\frac{1}{2}\left\|P_{-} t \psi\right\|^{2}-\int_{\Omega} a(x) G(t \psi) d x \\
= & \frac{1}{2}\left\|P_{+} t \psi\right\|^{2}-\frac{1}{2}\left\|P_{-} t \psi\right\|^{2}-\int_{\Omega^{+}} a^{+}(x) G(t \psi) d x+\int_{\Omega^{-}} a^{-}(x) G(t \psi) d x \\
\leq & \frac{1}{2}\left\|P_{+} t \psi\right\|^{2}-\frac{1}{2}\left\|P_{-} t \psi\right\|^{2}-\int_{\Omega^{+}} a^{+}(x)\left(a_{5} t^{\mu} \psi^{\mu}-a_{4}\right) d x \\
& +\|G(t \psi)\|_{\infty} \int_{\Omega^{-}} a^{-}(x) d x \\
\leq & \frac{1}{2} t^{2}-\int_{\Omega^{+}} a^{+}(x)\left(a_{5} t^{\mu} \psi^{\mu}-a_{4}\right) d x+\epsilon^{\prime}
\end{aligned}
$$

for small $\epsilon^{\prime}>0$. Since $\mu>2$, there exist $t_{n}$ great enough for each $n$ and an $R_{n}>0$ such that $u_{n}=t_{n} \psi$ and $I\left(u_{n}\right)<0$ if $u_{n} \in H_{n} \backslash B_{R_{n}}$ and $\left\|u_{n}\right\|>R_{n}$, so the lemma is proved. 
Let us set

$$
\Gamma_{n}=\left\{\gamma \in C([0,1], H) \mid \gamma(0)=0 \text { and } \gamma(1)=u_{n}\right\}
$$

and

$$
b_{n}=\inf _{\gamma \in \Gamma_{n}[0,1]} \max _{[0} I(\gamma(u)), \quad n \in N \text {. }
$$

Proof of Theorem 1.2(ii) We assume that $g(u) u-\mu G(u)$ is not bounded and there exists an $\epsilon>0$ such that $\int_{\Omega^{-}} a^{-}(x) d x<\epsilon$. By Proposition 2.1 and Proposition $2.2, I \in C^{1}(H, R)$ and satisfies the Palais-Smale condition. By Lemma 4.1, there exists an $R_{n}>0$ such that $I\left(u_{n}\right) \leq$ 0 for $u_{n} \in H_{n} \backslash B_{R_{n}}$. We note that $I(0)=0$. By Lemma 4.1 and the generalized mountain pass theorem, for $n$ large enough $b_{n}>0$ is a critical value of $I$ and $\lim _{n \rightarrow \infty} b_{n}=+\infty$. Let $\tilde{u}_{n}$ be a critical point of $I$ such that $I\left(\tilde{u}_{n}\right)=b_{n}$. Then for each real number $M, \max _{\Omega}\left|\tilde{u}_{n}(x)\right| \geq M$. In fact, by contradiction, $\Delta^{2} u+c \Delta u=a(x) g(u)$ and $\max _{\Omega}\left|\tilde{u}_{n}(x)\right| \leq K$ imply that

$$
I\left(\tilde{u}_{n}\right) \leq \max _{\left|\tilde{u}_{n}\right| \leq K}\left(\frac{1}{2} g\left(\tilde{u}_{n}\right) \tilde{u}_{n}-G\left(\tilde{u}_{n}\right)\right) \int_{\Omega}|a(x)| d x,
$$

which means that $b_{n}$ is bounded. This is absurd because of the fact that $\lim _{n \rightarrow \infty} b_{n}=+\infty$. Thus we complete the proof.

\section{Competing interests}

The authors declare that they have no competing interests.

\section{Authors' contributions}

TJ and Q-HC participated in the sequence alignment and drafted the manuscripted. Both authors read and approved the final manuscript.

\section{Author details}

${ }^{1}$ Department of Mathematics, Kunsan National University, Kunsan, 573-701, Korea. ${ }^{2}$ Department of Mathematics Education, Inha University, Incheon, 402-751, Korea.

\section{Acknowledgements}

This work (Choi) was supported by Basic Science Research Program through the National Research Foundation of Korea (NRF) funded by the Ministry of Education, Science and Technology (KRF-2013010343).

Received: 28 June 2013 Accepted: 8 January 2014 Published: 04 Feb 2014

\section{References}

1. Khanfir, S, Lassoued, L: On the existence of positive solutions of a semilinear elliptic equation with change of sign. Nonlinear Anal., Theory Methods Appl. 22(11), 1309-1314 (1994)

2. Choi, QH, Jung, T: Multiplicity results on nonlinear biharmonic operator. Rocky Mt. J. Math. 29(1), 141-164 (1999)

3. Jung, TS, Choi, QH: Multiplicity results on a nonlinear biharmonic equation. Nonlinear Anal., Theory Methods Appl. 30(8), 5083-5092 (1997)

4. Tarantello, G: A note on a semilinear elliptic problem. Differ. Integral Equ. 5(3), 561-565 (1992)

5. Micheletti, AM, Pistoia, A: Multiplicity results for a fourth-order semilinear elliptic problem. Nonlinear Anal. TMA 31(7), 895-908 (1998)

6. Choi, QH, Jung, T: Multiplicity of solutions and source terms in a fourth order nonlinear elliptic equation. Acta Math. Sci. 19(4), 361-374 (1999)

7. Rabinowitz, PH: Minimax Methods in Critical Point Theory with Applications to Differential Equations. CBMS Regional Conf. Ser. Math., vol. 65. Am. Math. Soc., Providence (1986) 